\title{
Between anchors and aspirations: a new family of bargaining solutions
}

\author{
Emin Karagözoğlu ${ }^{1,2}$ (D) Kerim Keskin ${ }^{3}$ Elif Özcan-Tok ${ }^{4}$
}

Received: 17 November 2018 / Accepted: 1 April 2019 / Published online: 5 April 2019

(c) Springer-Verlag GmbH Germany, part of Springer Nature 2019

\begin{abstract}
We study the salience and power of reference points in determining the effective anchors and aspirations in bargaining problems. Along this line, we enrich the analysis of the standard bargaining model with two new parameters: the first parameter can be interpreted as the effectiveness (or salience) of the reference point in determining the anchor, whereas the second parameter can be interpreted as its effectiveness in shaping agents' aspirations. Utilizing these parameters, we provide a unifying framework for the study of bargaining problems with a reference point. The two-parameter family of bargaining solutions we obtain encompasses some well-known solutions as special cases. We offer multiple characterizations for each individual member of this family as well as two characterizations for the whole solution family in bilateral bargaining problems.
\end{abstract}

Keywords Anchors - Aspirations - Axiomatic approach - Bargaining problems · Bargaining solutions · Disagreement point $\cdot$ Reference point

JEL Classification C72 · C78 · D63 · D74

\section{Introduction}

What provides a bargaining advantage? Nash (1950) proposed that what an agent would get in case of a disagreement may constitute a source of bargaining power/advantage. On the other hand, Gupta and Livne (1988) argued that reference points in the form of existing or expired contracts, precedents, negotiation text, or

Emin Karagözoğlu

karagozoglu@bilkent.edu.tr

1 Bilkent University, Ankara, Turkey

2 CESifo Munich, Munich, Germany

3 Kadir Has University, Istanbul, Turkey

4 Central Bank of the Republic of Turkey, Ankara, Turkey 
norms may also provide bargaining power. ${ }^{1}$ Most cooperative bargaining models employ the disagreement point (e.g., Nash 1950; Kalai and Smorodinsky 1975; Kalai 1977; Balakrishnan et al. 2011) or the reference point (e.g., Brito et al. 1977; Gupta and Livne 1988) as an anchor that influences the negotiated agreements. There is an important difference between the bargaining advantages provided by these two sources: the former can be exercised unilaterally (i.e., an agent would not need the opponent's permission to disagree) whereas the latter needs to be-at least tacitlymutually acknowledged (i.e., a reference point can be employed only if both parties find it sufficiently salient or reasonable). In that sense, the power provided by the disagreement point can be labeled as hard power, whereas the power provided by the reference point can be labeled as soft power (see Bolton and Karagözoğlu 2016). At first look, it appears that hard power should dominate soft power, when push comes to shove. But what if there is a well-established norm that implies a salient reference point? In other words, could it be that the effectiveness of hard power depends on the salience or the source of soft power?

The way disagreement and reference points are incorporated into most cooperative bargaining models implies that in addition to their direct effect mentioned above, they may also have an indirect effect on the negotiated agreement through their influence on agents' aspirations. Aspirations can be interpreted as agents' expectations on the best case scenario in negotiations. In other words, they provide answers to the question, "What is the most favorable outcome I can get out of this negotiation?". Kalai and Smorodinsky (1975) is among the first to argue that aspirations can influence agreements. ${ }^{2}$ In that study, agents' aspirations are directly derived from the disagreement point and the utility possibility frontier. Accordingly, the ideal point introduced by Kalai and Smorodinsky (1975) gives, for each agent, the maximum utility level that can be reached in an individually rational agreement. The Kalai-Smorodinsky solution employs the disagreement point (as an anchor point) and the ideal point (as an aspiration point) in proposing a settlement. On the other hand, the solution concept introduced by Gupta and Livne (1988) employs the reference point (as an anchor point) and the ideal point (as an aspiration point). Their model describes a bargaining situation where the salience of the reference point "increases the likelihood that no party exercises its threat to break off" (see Gupta and Livne 1988, p. 1304). Balakrishnan et al. (2011) introduced a new salient point into bargaining problems: the tempered aspirations point. Such aspirations are derived from the reference point instead of the disagreement point; and their solution-called the tempered aspirations solutionemploys the disagreement point (as an anchor point) and the tempered aspirations point (as an aspiration point). Their model describes a bargaining situation where "the salience of the reference point mutes or tempers the negotiators' aspirations" (see Balakrishnan et al. 2011, p. 144).

One can argue that anchor points describe what would happen in the worst case scenario, whereas aspiration points describe what would happen in each agent's indi-

\footnotetext{
1 A plethora of experimental studies provided evidence supporting these arguments. Among others, the reader is referred to Kristensen and Gärling (2000), Gächter and Riedl (2005, 2006), Anbarc1 and Feltovich (2013, 2018), Bartling and Schmidt (2015), Herweg and Schmidt (2013), Bolton and Karagözoğlu (2016), and Irlenbusch et al. (2017).

2 Some other studies that have similar arguments are Raiffa (1953) and Rosenthal (1976).
} 
vidual best case scenario; and that there are multiple candidates for both types of salient points which may lead to entirely different descriptions. What is common to all three solution concepts mentioned above is that each proposes a settlement as a feasible compromise between the worst case and the best case scenarios. A natural question is: In modeling a simple bargaining situation, why/when should the reference point be preferred over the disagreement point as an anchor point (as in the Kalai-Smorodinsky solution or the tempered aspirations solution), or vice versa (as in the Gupta-Livne solution)? Similarly, why/when should the ideal point be preferred over the tempered aspirations point as an aspiration point (as in the Kalai-Smorodinsky solution or the Gupta-Livne solution), or vice versa (as in the tempered aspirations solution)? As Balakrishnan et al. (2011, p. 149) correctly pointed out: "the context of the bargain will affect the manner in which the reference point influences the negotiated outcome".

To put it more concretely, below we provide some examples where the presence of a reference point likely has an impact on agents' bargaining behavior and the salience of the reference point (relative to the disagreement point) influences the magnitude of that impact. These examples are all inspired by the existing experimental research on the influences of reference/focal points and disagreement points on bargaining behavior and bargaining outcomes:

Example 1 (inspired by Bazerman 1985): Consider a wage bargaining such that the only piece of information the bargaining parties have is the payoffs they would receive if they cannot reach an agreement (Info 1). Now consider a variation where another piece of information is available, which is the average wage level in the same industry in the current year (Info 2). In the presence of this new information, which may likely act as a reference point, it is hard to believe that Info 1 will be as influential as before on the bargaining agreement. Finally, consider yet another variation where, instead of Info 2, the bargaining parties know the average wage level in a different industry from five years ago (Info $2^{\prime}$ ). It is likely that Info $2^{\prime}$ will be less influential on the bargaining agreement in the latter variation than Info 2 is in the former variation.

Example 2 (inspired by Gächter and Riedl 2005, 2006; and Karagözoğlu and Riedl 2015): Consider the following scenario borrowed from the experiments in Gächter and Riedl $(2005,2006)$. There is a hypothetical company where the top management paid salaries to two department heads based on their relative performances in the past. Now, things have changed, and the current department heads will bargain over the distribution of the total salary budget (after exerting effort in a real-effort task, which will determine their relative performances). They know that they would receive zero payoffs if they cannot reach an agreement (Info 1). Consider now two variations in which another piece of information is available: in Info 2, they are told that the top management implemented a 60-40 division in the past (favoring the higher performer); whereas in Info $2^{\prime}$ they are told that the top management implemented a 90-10 division in the past (favoring the higher performer). As in Example 1, this new information likely acts as a reference point influencing the bargaining agreement. Here, Info 2 arguably presents a more "reasonable" (or legitimate) reference point whereas Info 2 ' presents a less reasonable one due to its extreme asymmetry. One may guess that Info 2 will influence bargaining behavior and bargaining outcome, and it will do so more than Info $2^{\prime}$ would, which is, in fact, empirically validated by Gächter and Riedl (2005, 2006). 
Example 3 (inspired by Gächter and Riedl 2005; and Karagözoğlu and Riedl 2015): Consider a bargaining scenario where two individuals bargain over a pie of worth $\$ 100$, which is jointly produced by these individuals. The bargaining parties are aware that if no agreement is reached, they will receive certain disagreement payoffs (Info 1). Now, consider the following variations. In Info 2, individuals are informed about the average bargaining outcomes of bargaining instances where the respective bargaining parties jointly produced a surplus of $\$ 50$. In Info $2^{\prime}$, they are informed about the average bargaining outcomes of bargaining instances where a surplus of $\$ 50$ was exogenously given to the respective bargaining parties, as a result of a random draw. Arguably, for the bargaining situation at hand, the reference point in Info 2 is more salient (due to its similarity), hence will have a greater influence on the bargaining outcome compared to the reference point in Info 2'; a statement supported by the findings in Gächter and Riedl (2005) and Karagözoğlu and Riedl (2015).

Example 4 (inspired by Anbarc1 and Feltovich 2013, 2018): Consider another bargaining scenario where two individuals bargain over an exogenously given pie of worth $\$ 100$. The bargaining parties are aware that if no agreement is reached, each party $i$ will receive a payoff of $d_{i} \geq 0$. These disagreement payoffs can be very different from each other. We know that two prominent bargaining solutions, namely the Nash solution and the Kalai-Smorodinsky solution, propose that the disagreement payoffs directly and critically influence the bargaining outcome. That said, we also know that when the bargaining pie is exogenously given (as opposed to being jointly produced by the bargaining parties), the 50-50 division (i.e., equal division) likely acts as a reference point. In this particular scenario, its effectiveness may depend on the source of the disagreement payoffs. More precisely, Anbarcı and Feltovich (2013, 2018) and Feltovich (2019) showed that when the disagreement payoffs are earned (by exerting effort), then the 50-50 reference point/disagreement point becomes less/more effective in determining the bargaining outcome compared to the situation where the disagreement payoffs are randomly and exogenously assigned.

One common message from these examples is that it is very unlikely for a given axiomatic bargaining solution (e.g., the Nash solution or the Kalai-Smorodinsky solution) to make good predictions in rich bargaining contexts, where multiple factors (e.g., disagreement, ideal, and reference points) influence bargaining outcomes; and their influence, rather than being constant, can be a function of contextual factors. In fact, this is an empirically well-documented observation (see Fischer et al. 2007; Anbarc1 and Feltovich 2013, 2018; Bolton and Karagözoğlu 2016; Karagözoğlu and Kocher 2018; Feltovich 2019 among others). More precisely, the influence of a disagreement point on the negotiated outcome may depend on various factors such as how the disagreement point outcomes are determined, whether a salient reference point is present or not, and whether the allocation implied by the disagreement point is compatible with agents' fairness concerns or not. Similarly, the influence of a reference point on the negotiated outcome may depend on various factors such as similarity (between the current situation and the reference situation), temporality (whether the reference situation is contemporary or in the past), legitimacy, fairness, and time frame of negotiations. In a seminal paper, Sebenius (1992) discussed the need to incorporate contextual factors into negotiation analysis. Crusius et al. (2012) argued, by referring 
to some well-known experimental findings, that the context has an important influence on behavioral mechanisms and the effects of economic parameters. Along similar lines, it is argued by Yockey and Kruml (2009) and reported by Holm and Runnemark (2014) that the salience of a reference point is influenced by various contextual factors.

In this paper we argue that the salience of the reference point and its influence on agents' aspirations, respectively, provide new insights on the questions we posed above and has the potential to explain some regularities observed in bargaining experiments. Accordingly, we incorporate these two factors into the analysis of bargaining problems with a reference point. In particular, we introduce two parameters, $\alpha \in[0,1]$ and $\beta \in[0,1]$, which capture the salience of the reference point in determining the anchor and its influence on agents' aspirations, respectively. A higher value of $\alpha$ refers to a higher influence on the anchor whereas a higher value of $\beta$ refers to a higher influence on agents' aspirations. This gives us a rich and unifying framework for the study of bargaining problems with a reference point. It can be argued that our paper offers at least a partial answer to the critique by Roemer (1986) - an answer that still relies on the axiomatic bargaining theory. ${ }^{3}$

These two parameters also allow us to obtain a new family of bargaining solutions. This family encompasses some of the well-known solution concepts as special (corner) cases. For instance, when $\alpha=0$ and $\beta=0$ (i.e., the reference point has no influence on the anchor point or the aspiration point), this solution coincides with the KalaiSmorodinsky solution. When $\alpha=1$ and $\beta=0$ (i.e., the reference point completely determines the anchor point but has no influence on the aspiration point), this solution coincides with the Gupta-Livne solution. When $\alpha=0$ and $\beta=1$ (i.e., the reference point has no influence on the anchor point but completely determines the aspiration point), this solution coincides with the tempered aspirations solution. Finally, when $\alpha=1$ and $\beta=1$ (i.e., the reference point completely determines both the anchor point and the aspiration point), this solution coincides with what Gupta and Livne (1989) called the local Kalai-Smorodinsky solution. Naturally, in between these four extreme cases, there are infinitely many intermediate solution concepts that propose settlements by offering feasible compromises between the worst case and the best case scenarios described by anchors and aspirations, respectively. In this paper, we offer multiple characterizations for each individual member of the $(\alpha, \beta)$-family as well as two characterizations for the whole solution family.

Our work shares a similar spirit with Thomson (1981), Anbarc1 (1995), Alós-Ferrer et al. (2018), and Haake and Qin (2018) in that these papers also studied reference points and/or classes of bargaining solutions. Thomson (1981) introduced the concept of a reference function, which determines a reference point for each bargaining problem - as a function of the problem. Later, using the reference function concept of Thomson (1981), Anbarc1 (1995) proposed a class of solutions the outcomes of which lead to balanced concessions. The Kalai-Smorodinsky solution appears to be a special case of this class. Recently, Alós-Ferrer et al. (2018) introduced the negotiated aspirations solution that proposes the best allocation in the direction of the ideal point starting at an endogenous reference point. Their reference point is endogenous

\footnotetext{
3 Roemer (1986) argued that the axiomatic bargaining model is too impoverished and as such it fails to capture important issues in distributive justice.
} 
in that it is a function of the ideal point and the bargaining power. Finally, Haake and Qin (2018) introduced the constant elasticity of substitution (CES) family of bargaining solutions, which admits the Nash solution and the egalitarian solution as special cases. They also studied a normalized version of this family, which admits the Nash solution and the Kalai-Smorodinsky solution as special cases. Just as we do in the current paper, the authors provided unified characterizations of existing as well as new bargaining solutions.

The roadmap for the rest of this paper is as follows: Sect. 2 introduces the bargaining problem with a reference point and the $(\alpha, \beta)$-family of bargaining solutions. Section 3 includes an inventory of axioms used in the characterization results that follow. Section 4.1 presents characterizations of the individual members of the $(\alpha, \beta)$-family, Sect. 4.2 presents characterizations of the whole $(\alpha, \beta)$-family, Sect. 4.3 presents a comparative statics analysis, and Sect. 4.4 presents discussions and further remarks. Finally, Sect. 5 concludes with some limitations and possible future research.

\section{The model}

An $n$-person bargaining problem with a reference point is a triple $(S, d, r)$ where $S$ denotes the set of feasible outcomes, $d$ is the disagreement point, and $r$ is the reference point. We assume that (i) $S \subset \mathbb{R}^{n}$ is a non-empty, closed, convex, and comprehensive set; (ii) $\exists p \in \mathbb{R}_{++}^{n}, \exists q \in \mathbb{R}$ such that $\forall x \in S: \sum_{i} p_{i} x_{i} \leq q$; (iii) $d, r \in S$; (iv) $\exists x \in S$ with $x>d$; and (v) $r \geq d .^{4,5}$ Let $\mathfrak{a}(S, x)$ denote the aspiration vector such that for every $i \in\{1, \ldots, n\}$ and every $x \in S: \mathfrak{a}_{i}(S, x) \equiv \max \left\{t \in \mathbb{R} \mid\left(t, x_{-i}\right) \in S\right\}$. Accordingly, $\mathfrak{a}(S, d)$ is the ideal point (see Kalai and Smorodinsky 1975) and $\mathfrak{a}(S, r)$ is the tempered aspirations point (see Balakrishnan et al. 2011).

Let $\Sigma^{n}$ be the class of all bargaining problems with a reference point. A solution concept for such problems is a function $F: \Sigma^{n} \rightarrow \mathbb{R}^{n}$ that associates each $(S, d, r) \in$ $\Sigma^{n}$ with a unique point of $S$. Below, we present the definitions of some solution concepts we will use in the remainder of the paper.

Definition 1 (Kalai-Smorodinsky Solution) For every $(S, d, r) \in \Sigma^{n}$,

$$
K S(S, d, r)=\lambda^{*} \mathfrak{a}(S, d)+\left(1-\lambda^{*}\right) d
$$

where $\lambda^{*}=\max \{\lambda \in[0,1] \mid \lambda \mathfrak{a}(S, d)+(1-\lambda) d \in S\}{ }^{6}$

\footnotetext{
${ }^{4}$ For $x, y \in \mathbb{R}^{n}$, the vector inequalities are given by: $x \geq y, x>y$, and $x \gg y$.

5 The convexity assumption indicates that agents could agree to take a coin toss between two outcomes and that each agent's payoff from the coin toss is the average of his/her payoffs from these outcomes. Closedness of $S$ means that the set of physical agreements is closed and that agents' payoff functions are continuous. Comprehensiveness means that if $x \in S$, then for every $y \in \mathbb{R}_{+}^{n}$ with $y \leq x: y \in S$, which stipulates that utility is freely disposable. The assumption $d \in S$ indicates that agents are able to agree to disagree, the assumption $r \in S$ indicates that the reference point is feasible, and the assumption $r \geq d$ indicates that the reference point is individually rational. By assuming that there exists $x \in S$ with $x>d$, we rule out degenerate problems in which no agreement can make all agents better-off than the disagreement outcome. Finally, the condition (ii) implies the boundedness of $S$ from above, which means that the maximum payoff an agent can achieve out of an agreement is finite.

${ }^{6}$ Note that the Kalai-Smorodinsky solution does not depend on the reference point and is usually defined on $(S, d)$. Nevertheless, it is mathematically not problematic to define it on $(S, d, r)$.
} 
The Kalai-Smorodinsky solution proposes the maximum point of the bargaining set on the line segment connecting the ideal point, $\mathfrak{a}(S, d)$, and the disagreement point, $d$ (see Fig. 2a).

Definition 2 (Gupta-Livne Solution) For every $(S, d, r) \in \Sigma^{n}$,

$$
G L(S, d, r)=\lambda^{*} \mathfrak{a}(S, d)+\left(1-\lambda^{*}\right) r
$$

where $\lambda^{*}=\max \{\lambda \in[0,1] \mid \lambda \mathfrak{a}(S, d)+(1-\lambda) r \in S\}$.

The Gupta-Livne solution proposes the maximum point of the bargaining set on the line segment connecting the ideal point, $\mathfrak{a}(S, d)$, and the reference point, $r$ (see Fig. 2b).

Definition 3 (Tempered Aspirations Solution) For every $(S, d, r) \in \Sigma^{n}$,

$$
T A(S, d, r)=\lambda^{*} \mathfrak{a}(S, r)+\left(1-\lambda^{*}\right) d
$$

where $\lambda^{*}=\max \{\lambda \in[0,1] \mid \lambda \mathfrak{a}(S, r)+(1-\lambda) d \in S\}$.

The tempered aspirations solution proposes the maximum point of the bargaining set on the line segment connecting the tempered aspirations point, $\mathfrak{a}(S, r)$, and the disagreement point, $d$ (see Fig. 2c).

Definition 4 (Local Kalai-Smorodinsky Solution) For every $(S, d, r) \in \Sigma^{n}$,

$$
L K S(S, d, r)=\lambda^{*} \mathfrak{a}(S, r)+\left(1-\lambda^{*}\right) r
$$

where $\lambda^{*}=\max \{\lambda \in[0,1] \mid \lambda \mathfrak{a}(S, r)+(1-\lambda) r \in S\}$.

The local Kalai-Smorodinsky solution proposes the maximum point of the bargaining set on the line segment connecting the tempered aspirations point, $\mathfrak{a}(S, r)$, and the reference point, $r$ (see Fig. $2 \mathrm{~d}$ ).

As discussed above, we introduce two new parameters in this paper: $\alpha \in[0,1]$, which can be interpreted as the influence of the reference point in determining the anchor (or, simply, its salience); and $\beta \in[0,1]$, which can be interpreted as the influence of the reference point in shaping agents' aspirations/expectations. Accordingly, for any $\alpha, \beta \in[0,1]$, we define the $(\alpha, \beta)$-solution as follows.

Definition 5 For a given $\alpha, \beta \in[0,1]$ and for every $(S, d, r) \in \Sigma^{n}$,

$$
F^{\alpha, \beta}(S, d, r)=\lambda^{*} \mathfrak{a}(S, \beta r+(1-\beta) d)+\left(1-\lambda^{*}\right)(\alpha r+(1-\alpha) d)
$$

where $\lambda^{*}=\max \{\lambda \in[0,1] \mid \lambda \mathfrak{a}(S, \beta r+(1-\beta) d)+(1-\lambda)(\alpha r+(1-\alpha) d) \in S\}$.

For every $\alpha, \beta \in[0,1]$, the $(\alpha, \beta)$-solution proposes the maximum point of the bargaining set on the line segment connecting $\mathfrak{a}(S, \beta r+(1-\beta) d)$ and $\alpha r+(1-\alpha) d$ (see Fig. 1). The collection of all such solutions constitutes the $(\alpha, \beta)$-family of bargaining solutions. 


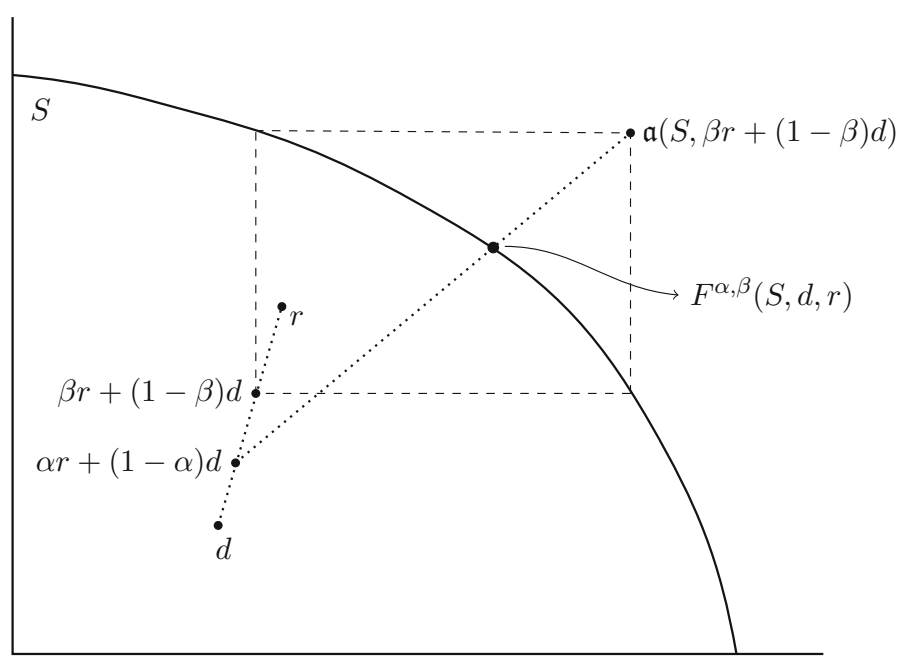

Fig. 1 The $(\alpha, \beta)$-solution

As depicted in Fig. 2, when $(\alpha, \beta)=(0,0)$, the $(\alpha, \beta)$-solution coincides with the Kalai-Smorodinsky solution. This indicates that the Kalai-Smorodinsky solution implicitly assumes that even when there is a reference point in the environment, it is completely ineffective in determining the anchor or aspiration points. When $(\alpha, \beta)=(0,1)$, the $(\alpha, \beta)$-solution coincides with the tempered aspirations solution, which shows that the tempered aspirations solution assumes that the reference point is completely effective in shaping the aspiration point, but has no influence on the anchor point. When $(\alpha, \beta)=(1,0)$, it coincides with the Gupta-Livne solution, which shows that the Gupta-Livne solution assumes that the reference point is completely effective in determining the anchor point, but has no influence on the aspiration point. Finally, when $(\alpha, \beta)=(1,1)$, it coincides with the local Kalai-Smorodinsky solution. This indicates that the local Kalai-Smorodinsky solution assumes that the reference point is completely effective in determining both the anchor and aspiration points. Between these corner cases, the $(\alpha, \beta)$-solution family encompasses all other solution concepts of similar sort. In other words, our model introduces a rich apparatus that can allow researchers to incorporate contextual factors and their influences on anchors and aspirations to the investigation of bargaining problems.

Note that our model allows $\alpha$ and $\beta$ to be equal, but simply does not only consider this very special case. To the extent that anchors and aspirations are salient points of different nature, this is a natural modeling assumption. Accordingly, we offer a richer description of the bargaining context compared to the one that restricts attention to cases where $\alpha$ and $\beta$ are equal. 


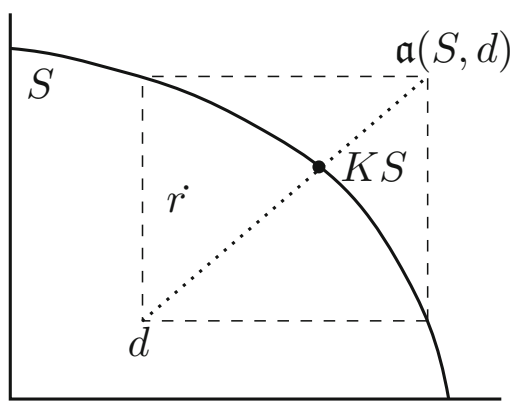

(a) Kalai-Smorodinsky Solution

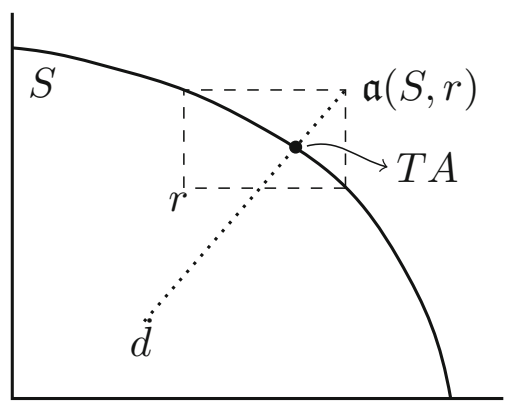

(c) Tempered Aspirations Solution

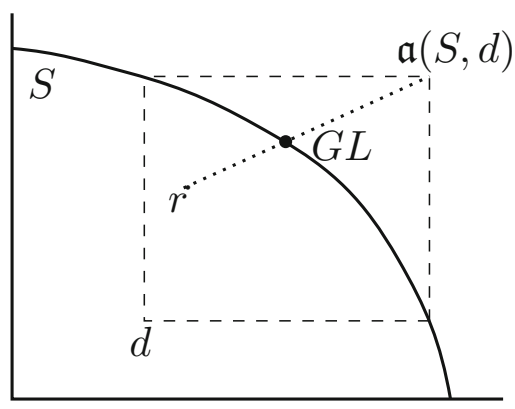

(b) Gupta-Livne Solution

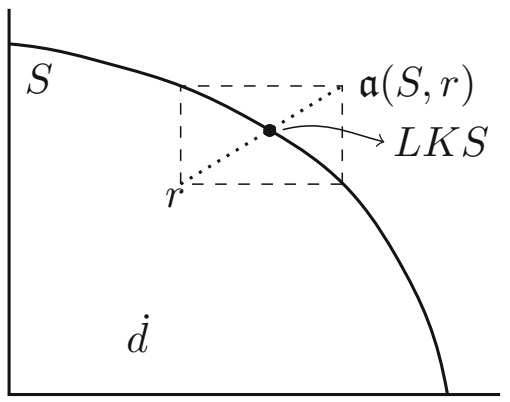

(d) Local Kalai-Smorodinsky Solution

Fig. 2 Four bargaining solutions as members of the $(\alpha, \beta)$-family

\section{Inventory of axioms}

In the following, we present the definitions of the axioms employed in our characterizations. Since the characterization results concern bilateral bargaining problems, these definitions are also given for bilateral bargaining problems.

First, for every $S \subset \mathbb{R}^{2}$, we define the set of weakly Pareto optimal outcomes as $W P O(S)=\{x \in S \mid \nexists y \in S \backslash\{x\}: y \gg x\}$ and the set of Pareto optimal outcomes as $P O(S)=\{x \in S \mid \nexists y \in S \backslash\{x\}: y>x\}$. The following standard axioms require the solution to be (weakly) Pareto optimal.

Axiom 1 (Weak Pareto Optimality)(WPO) For every $(S, d, r) \in \Sigma^{2}, F(S, d, r) \in$ $W P O(S)$.

Axiom 2 (Pareto Optimality)(PO) For every $(S, d, r) \in \Sigma^{2}, F(S, d, r) \in P O(S)$.

Let $T_{\text {sym }}: \mathbb{R}^{2} \rightarrow \mathbb{R}^{2}$ be a transformation such that $T_{\text {sym }}\left(x_{1}, x_{2}\right)=\left(x_{2}, x_{1}\right)$. Also let $T_{\text {sym }}(S)=\left\{T_{\text {sym }}(x) \mid x \in S\right\}$. The following is a primitive fairness axiom, which is standard in the literature on bargaining problems.

Axiom 3 (Symmetry)(SYM) For every $(S, d, r) \in \Sigma^{2}, F\left(T_{\text {sym }}(S), T_{\text {sym }}(d), T_{\text {sym }}(r)\right)$ $=T_{\text {sym }}(F(S, d, r))$. 
A bargaining problem $(S, d, r) \in \Sigma^{2}$ is said to be symmetric if $T_{\text {sym }}(S)=S$, $T_{\text {sym }}(d)=d$, and $T_{\text {sym }}(r)=r$. For such a problem, if a bargaining solution $F$ : $\Sigma^{2} \rightarrow \mathbb{R}^{2}$ satisfies SYM, then $F_{1}(S, d, r)=F_{2}(S, d, r)$.

For the following axiom, we say that $T_{a f f} \equiv\left(T_{a f f, 1}, T_{a f f, 2}\right): \mathbb{R}^{2} \rightarrow \mathbb{R}^{2}$ is a positive affine transformation if for each $i \in\{1,2\}$, the map $T_{a f f, i}\left(x_{1}, x_{2}\right)$ is of the form $m_{i} x_{i}+n_{i}$ for some positive constant $m_{i}$ and another constant $n_{i}$. We also define $T_{a f f}(S)=\left\{T_{a f f}(x) \mid x \in S\right\}$. The axiom requires the solution to be invariant under positive affine transformations of a given problem.

Axiom 4 (Invariance Under Positive Affine Transformations)(IPAT) For every $(S, d, r) \in \Sigma^{2}, F\left(T_{a f f}(S), T_{a f f}(d), T_{a f f}(r)\right)=T_{a f f}(F(S, d, r))$.

In Sect. 4.1, we provide characterization results for the individual members of the $(\alpha, \beta)$-family. In this regard, we must have axiom families rather than single axioms (e.g., Axioms 5-9). This way, there would be a one-to-one mapping between the solution family and each axiom family.

To understand the common intuition underlying the following axioms, assume that there is an arbitrator who wants to resolve a conflict in a given bargaining problem and has certain normative concerns. Given the bargaining context, the arbitrator also has an opinion on the (effective) anchor point and the (effective) aspiration point to be employed: $a r+(1-a) d$ and $\mathfrak{a}(S, b r+(1-b) d)$, respectively. Such an arbitrator would naturally consider axioms that use these salient points rather than axioms using the disagreement point and/or the reference point. Moreover, note that Axioms 5-9 are generalizations of the corresponding monotonicity, sensitivity, and relevance axioms in earlier work (see Kalai and Smorodinsky 1975; Gupta and Livne 1988; Balakrishnan et al. 2011). Naturally, they have identical interpretations and normative appeal with those axioms.

The following is a monotonicity axiom, which is analogous to individual monotonicity introduced in Kalai and Smorodinsky (1975). It stipulates that an expansion of the bargaining set, which improves agent $i$ 's aspiration point and keep agent $j$ 's aspiration point the same, should not make agent $i$ worse off.

Axiom 5 (Individual ( $a, b)$-Monotonicity)(IND ( $a, b)$-MON) Take any $(S, d, r)$, $\left(S^{\prime}, d^{\prime}, r^{\prime}\right) \in \Sigma^{2}$ such that for some $j \in\{1,2\}: \mathfrak{a}_{j}(S, b r+(1-b) d)=$ $\mathfrak{a}_{j}\left(S^{\prime}, b r^{\prime}+(1-b) d^{\prime}\right)$ and for $i \neq j: \mathfrak{a}_{i}(S, x) \leq \mathfrak{a}_{i}\left(S^{\prime}, x\right)$ for every $x \in S$. If $a r+(1-a) d=a r^{\prime}+(1-a) d^{\prime}$, then $F_{i}(S, d, r) \leq F_{i}\left(S^{\prime}, d^{\prime}, r^{\prime}\right)$.

The following axiom stipulates that if the bargaining set expands in such a way that there is no change in any salient point, then no agent will be worse off. The intuition is that a bargaining set expansion, which does not influence agents' bargaining powers (i.e., anchors or aspirations), is collectively good since a larger set means more alternatives.

Axiom 6 (b-Restricted ( $a, b)$-Monotonicity)(b-REST ( $a, b)$-MON) Take any $(S, d, r)$, $\left(S^{\prime}, d^{\prime}, r^{\prime}\right) \in \Sigma^{2}$ such that $S \subset S^{\prime}$, ar $+(1-a) d=a r^{\prime}+(1-a) d^{\prime}$, and $b r+(1-b) d=b r^{\prime}+(1-b) d^{\prime}$. If $\mathfrak{a}(S, b r+(1-b) d)=\mathfrak{a}\left(S^{\prime}, b r^{\prime}+(1-b) d^{\prime}\right)$, then $F(S, d, r) \leq F\left(S^{\prime}, d^{\prime}, r^{\prime}\right)$. 
The following axiom indicates that given $a, b \in[0,1]$, if the corresponding point from which aspirations are derived is trivial (i.e., ineffective), then the situation can be represented by a reduced problem in which the reference point and the disagreement point coincide. Simply put, the point from which aspirations are derived does not have a stand-alone importance: if it does not lead to an aspiration point different from the one that would be derived using the anchor point, then one can focus on a more concisely described bargaining problem.

Axiom 7 (Reduction under Trivial ( $a, b)$-Salient Points)(REDT ( $a, b)$-SP) For every $(S, d, r) \in \Sigma^{2}$, if $\mathfrak{a}(S, a r+(1-a) d)=\mathfrak{a}(S, b r+(1-b) d)$, then $F(S, d, r)=$ $F(S, a r+(1-a) d, a r+(1-a) d)$.

The following axiom requires that if the bargaining problem changes in such a way that the point from which aspirations are derived is the only change, then the solution will not be affected. The intuition is that the changes in the point from which aspirations are derived influences the bargaining outcome to the extent that it influences the aspiration point: if such a change does not affect the aspiration point, then it should not influence the bargaining outcome.

Axiom 8 (Limited Sensitivity to Changes in the ( $a, b)$-Salient Point)(LSC $(a, b)-S P)$ For every $(S, d, r),\left(S^{\prime}, d^{\prime}, r^{\prime}\right) \in \Sigma^{2}$, if $S=S^{\prime}$, ar $+(1-a) d=a r^{\prime}+(1-a) d^{\prime}$, and $\mathfrak{a}(S, b r+(1-b) d)=\mathfrak{a}\left(S^{\prime}, b r^{\prime}+(1-b) d^{\prime}\right)$, then $F(S, d, r)=F\left(S^{\prime}, d^{\prime}, r^{\prime}\right)$.

The following is another monotonicity axiom, which is weaker than Axiom 5 and stronger than Axiom 6.

Axiom 9 (Restricted ( $a, b)$-Monotonicity)(REST ( $a, b)$-MON) For every $(S, d, r)$, $\left(S^{\prime}, d^{\prime}, r^{\prime}\right) \in \Sigma^{2}$, if $S \subset S^{\prime}, a r+(1-a) d=a r^{\prime}+(1-a) d^{\prime}$, and $\mathfrak{a}(S, b r+(1-b) d)=$ $\mathfrak{a}\left(S^{\prime}, b r^{\prime}+(1-b) d^{\prime}\right)$, then $F(S, d, r) \leq F\left(S^{\prime}, d^{\prime}, r^{\prime}\right)$.

Finally, we present two additional axioms, which are weaker versions of Axiom 5 and Axiom 9, respectively. These axioms will be employed in Sect. 4.2 when we present characterizations of the whole $(\alpha, \beta)$-family.

Axiom 10 (Weak Individual Monotonicity)(WEAK IND MON) A bargaining solution $F: \Sigma^{2} \rightarrow \mathbb{R}^{2}$ satisfies WEAK IND MON, if it satisfies IND $(a, b)$-MON for some $(a, b) \in[0,1]^{2}$.

Axiom 11 (Weak Restricted Monotonicity)(WEAK REST MON) A bargaining solution $F: \Sigma^{2} \rightarrow \mathbb{R}^{2}$ satisfies WEAK REST MON, if it satisfies REST $(a, b)$-MON for some $(a, b) \in[0,1]^{2}$.

\section{The results}

In this section, we provide multiple characterizations of the individual members of the $(\alpha, \beta)$-family in bilateral bargaining problems. Stemming from two of these characterizations, we further obtain two independent characterizations of the whole family. Finally, we present comparative statics results and some further remarks. 


\subsection{Characterizations of individual $(\alpha, \beta)$-solutions}

We start by analyzing the relations between the axiom families introduced in the previous section. In the following lemmas, we show that IND $(a, b)$-MON implies REST $(a, b)$-MON; that REST $(a, b)$-MON implies $b$-REST $(a, b)$-MON and LSC $(a, b)$-SP; and that LSC $(a, b)$-SP implies REDT $(a, b)$-SP. All proofs are relegated to the Appendix.

Lemma 1 For a given $(a, b) \in[0,1]^{2}$, any bargaining solution $F: \Sigma^{2} \rightarrow \mathbb{R}^{2}$ that satisfies IND $(a, b)$-MON also satisfies REST $(a, b)$-MON.

Lemma 2 For a given $(a, b) \in[0,1]^{2}$, any bargaining solution $F: \Sigma^{2} \rightarrow \mathbb{R}^{2}$ that satisfies REST ( $a, b)$-MON also satisfies b-REST $(a, b)$-MON.

Lemma 3 For a given $(a, b) \in[0,1]^{2}$, any bargaining solution $F: \Sigma^{2} \rightarrow \mathbb{R}^{2}$ that satisfies REST $(a, b)$-MON also satisfies LSC $(a, b)-S P$.

Lemma 4 For a given $(a, b) \in[0,1]^{2}$, any bargaining solution $F: \Sigma^{2} \rightarrow \mathbb{R}^{2}$ that satisfies LSC $(a, b)$-SP also satisfies REDT $(a, b)-S P$.

Theorem 1 below presents multiple characterizations of individual members of the $(\alpha, \beta)$-family.

Theorem 1 For a given $(a, b) \in[0,1]^{2}$, a bargaining solution $F: \Sigma^{2} \rightarrow \mathbb{R}^{2}$ is the $(a, b)$-solution if and only if $F$ satisfies

(i) WPO, SYM, IPAT, and IND $(a, b)-M O N$;

(ii) WPO, SYM, IPAT, and REST ( $a, b)-M O N$;

(iii) WPO, SYM, IPAT, $b$-REST (a,b)-MON, and LSC $(a, b)-S P$.

Moreover, if $a \leq b$, then $F: \Sigma^{2} \rightarrow \mathbb{R}^{2}$ is the $(a, b)$-solution if and only if $F$ satisfies

(iv) WPO, SYM, IPAT, $b$-REST $(a, b)-M O N$, and REDT $(a, b)-S P$.

Proof See the Appendix.

By Lemmas 1-4, we see that the characterizations in Theorem 1 are given in an ascending order of tightness. Therefore, if one compares our characterizations using tightness as measure, one prefers (iii) to (ii) and (ii) to (i); and also (iv) to all others if $a \leq b$. Yet, we think that each characterization has a value since they allow us to describe and identify the members of the $(\alpha, \beta)$-family with different characteristics.

Furthermore, when $(a, b)=(0,0)$, parts $(i i),(i i i)$, and (iv) of Theorem 1 become alternative characterizations for the Kalai-Smorodinsky solution; when $(a, b)=$ $(1,0)$, parts $(i)$ and $(i i)$ become alternative characterizations for the Gupta-Livne solution; and when $(a, b)=(0,1)$, parts $(i)$, (ii), and (iii) become alternative characterizations for the tempered aspirations solution.

Finally, Fig. 3 summarizes the relations between the axioms used in our characterizations and how they characterize the $(a, b)$-solution. ${ }^{7}$

\footnotetext{
7 Assume that the numbers in the sets denote the name of the sets. The figure utilizes the facts that $(i)$ $2 \subset 3$ by Lemma 1; (ii) $3 \subset 4$ by Lemma 2 ; and (iii) $3 \subset 5 \subset 6$ by Lemmas $3-4$.
} 


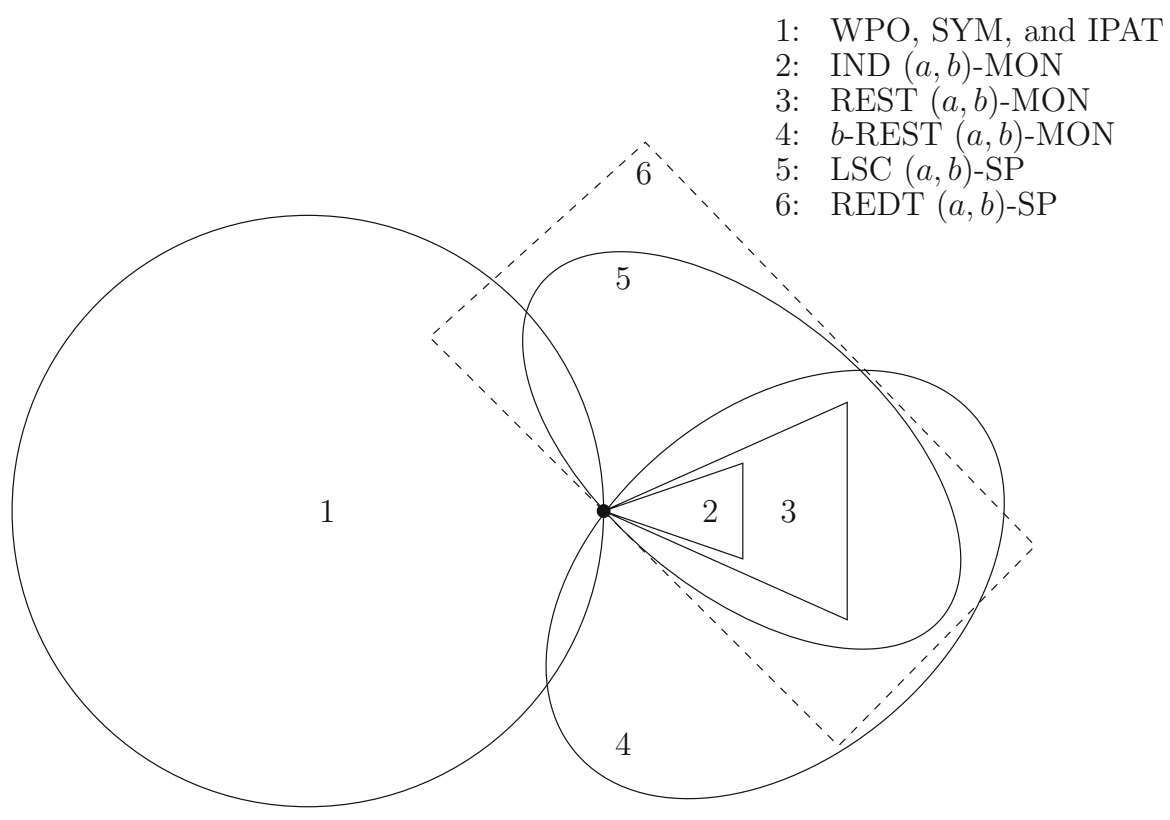

Fig. 3 The summary of the characterization results

\subsection{Characterizations of the $(\alpha, \beta)$-family}

In the previous subsection, we provided multiple characterizations of individual members of the $(\alpha, \beta)$-family. Here, we provide two independent characterization results for the whole $(\alpha, \beta)$-family.

Theorem 2 A bargaining solution $F: \Sigma^{2} \rightarrow \mathbb{R}^{2}$ is a member of the $(\alpha, \beta)$-family if and only if $F$ satisfies

(i) WPO, SYM, IPAT, and WEAK IND MON;

(ii) WPO, SYM, IPAT, and WEAK REST MON.

Proof See the Appendix.

In the light of our characterization results, the arbitrator interpretation for bargaining solutions leads to a new insight: Given the bargaining context, $\alpha$ and $\beta$ can be thought of as describers of the arbitrator's opinion on the most appropriate monotonicity axiom from the corresponding axiom family. The arbitrator may believe that a "good" solution should satisfy the monotonicity requirement for some $a, b \in[0,1]$. Theorem 2 implies that such an arbitrator would prefer a member of the $(\alpha, \beta)$-family. Moreover, if the arbitrator thinks that the (effective) salient points are $a^{*} r+\left(1-a^{*}\right) d$ and $\mathfrak{a}\left(S, b^{*} r+\right.$ $\left.\left(1-b^{*}\right) d\right)$, so that "the best" solution should satisfy the monotonicity requirement for $\left(a^{*}, b^{*}\right)$, then Theorem 1 suggests that the only solution that could be employed by such an arbitrator is the $\left(a^{*}, b^{*}\right)$-solution.

At this point, we revisit some of the examples mentioned in the Introduction. In Example 1, what the bargaining parties would receive if they cannot reach an agreement 
can be represented by the disagreement point, $d$. When the arbitrator is informed about a previous wage agreement, he might employ that particular agreement as the reference point, $r$. In case the previous agreement is from the same industry in the current year, the arbitrator will likely have the opinion that the reference point should be very influential in determining the salient points, employing high values of $a$ and $b$; whereas if the previous agreement is from a different industry five years ago, the arbitrator will likely have the opinion that the reference point should not have much effect on the salient points, employing low values of $a$ and $b$. Similarly, in Example 3, assuming that there is an arbitrator, he would make use of the average bargaining outcome information in reaching a decision, but he would place a higher weight on it in case the data come from interactions where the surplus was jointly produced (as in the current problem in his hand).

\subsection{Comparative statics}

It is important to understand how the bargaining outcome changes in response to changes in the two parameters, $\alpha$ and $\beta$. To answer this question, relying on the IPAT axiom, we consider a bilateral bargaining problem $(S, d, r)$ where $d=0$ and the highest achievable utility for each player is 1 . Given any reference point $r=\left(r_{1}, r_{2}\right)$, for any $(a, b)$-solution from the $(\alpha, \beta)$-family, the bargaining outcome $\left(x_{1}^{*}, x_{2}^{*}\right)$ is defined to be on the bargaining frontier as well as on the line segment connecting $\left(a r_{1}, a r_{2}\right)$ and $\left(\mathfrak{a}_{1}\left(S, b r_{2}\right), \mathfrak{a}_{2}\left(S, b r_{1}\right)\right)$. The latter implies that $\left(x_{1}^{*}, x_{2}^{*}\right)$ solves the equation

$$
x_{2}^{*}=\frac{\mathfrak{a}_{2}\left(S, b r_{1}\right)-a r_{2}}{\mathfrak{a}_{1}\left(S, b r_{2}\right)-a r_{1}} x_{1}^{*}-\frac{a\left(\mathfrak{a}_{1}\left(S, b r_{2}\right) r_{2}-\mathfrak{a}_{2}\left(S, b r_{1}\right) r_{1}\right)}{\mathfrak{a}_{1}\left(S, b r_{2}\right)-a r_{1}} .
$$

Now, if there is an increase from $a$ to $a^{\prime}$ in the parameter $\alpha$, the respective change is illustrated in Fig. $4 a{ }^{8}$ Analytically, consider the slope of the line given in equation (1). Taking the derivative with respect to $a$ yields

$$
\frac{\mathfrak{a}_{2}\left(S, b r_{1}\right) r_{1}-\mathfrak{a}_{1}\left(S, b r_{2}\right) r_{2}}{\left(\mathfrak{a}_{1}\left(S, b r_{2}\right)-a r_{1}\right)^{2}}
$$

This derivative turns out to be negative when its numerator is negative, which happens if $\mathfrak{a}_{2}\left(S, b r_{1}\right) r_{1}<\mathfrak{a}_{1}\left(S, b r_{2}\right) r_{2}$, i.e., if the reference point and the bargaining frontier favor agent 2 more than they favor agent 1 ; and if so, as $a$ increases, we know that the slope of equation (1) decreases. Assume that this is the case, consider $a^{\prime}>a$; and write equation $\left(1^{\prime}\right)$ by replacing $a$ with $a^{\prime}$ in equation (1). Since both lines pass from $\left(\mathfrak{a}_{1}\left(S, b r_{2}\right), \mathfrak{a}_{2}\left(S, b r_{1}\right)\right)$ and equation (1) is relatively steeper, and given the shape of the bargaining frontier, we conclude that the $\left(a^{\prime}, b\right)$-solution yields a higher share to agent 2 compared to the $(a, b)$-solution. If otherwise, the converse result follows.

\footnotetext{
8 The calligraphic letters, $\mathcal{A}, \mathcal{A}^{\prime}, \mathcal{B}, \mathcal{B}^{\prime}$ in Figs. $4 \mathrm{a}$, b refer to the effective anchor points and the effective points from which aspirations are derived. We refrained from writing them explicitly since those multiple, long expressions would have congested the graphs.
} 


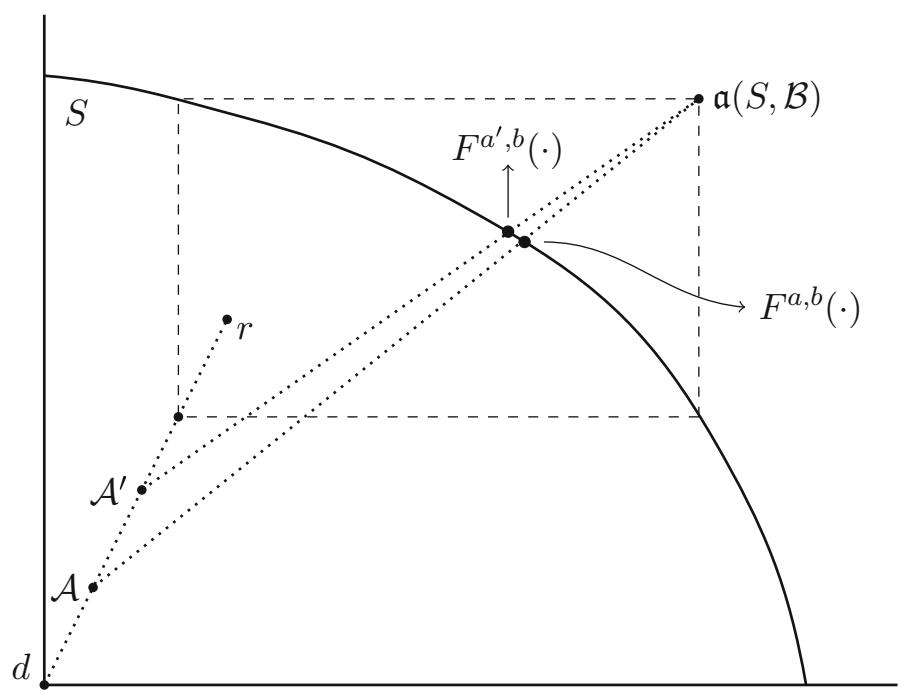

(a) Responses to Changes in $\alpha$

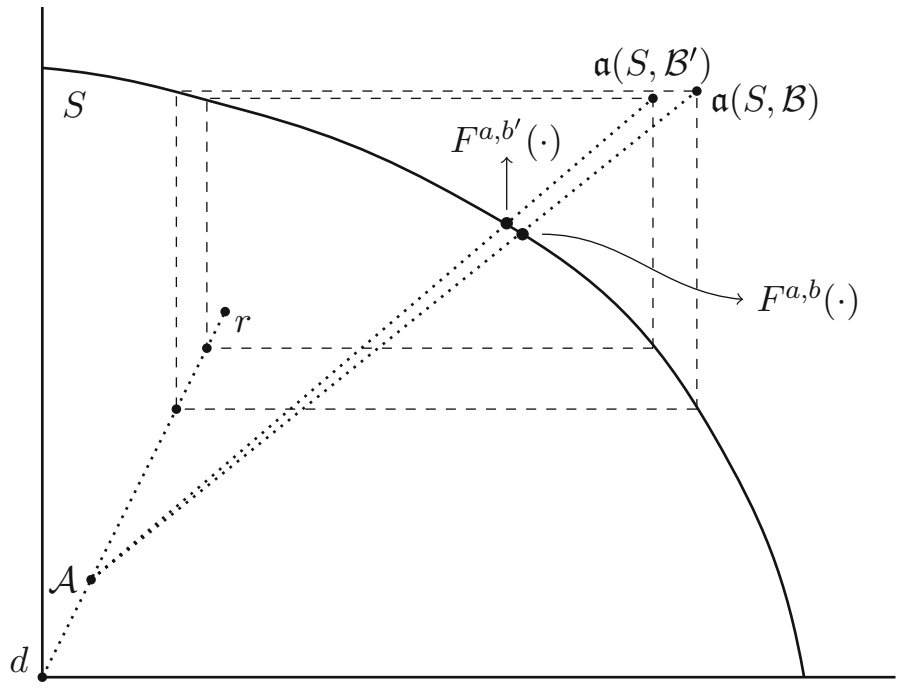

(b) Responses to Changes in $\beta$

Fig. 4 The illustration of the comparative statics results

Furthermore, Fig. 4b illustrates an increase from $b$ to $b^{\prime}$ in the parameter $\beta$. Assuming that $\mathfrak{a}_{1}$ and $\mathfrak{a}_{2}$ are differentiable in their second arguments, ${ }^{9}$ taking the derivative of the same slope with respect to $b$ yields

\footnotetext{
9 If the function representing the bargaining frontier is not differentiable at some $x_{1}$ or $x_{2}$, then these functions would not be differentiable at that point either.
} 


$$
\frac{\frac{\partial \mathfrak{a}_{2}\left(S, b r_{1}\right)}{\partial b}\left(\mathfrak{a}_{1}\left(S, b r_{2}\right)-a r_{1}\right)-\frac{\partial \mathfrak{a}_{1}\left(S, b r_{2}\right)}{\partial b}\left(\mathfrak{a}_{2}\left(S, b r_{1}\right)-a r_{2}\right)}{\left(\mathfrak{a}_{1}\left(S, b r_{2}\right)-a r_{1}\right)^{2}}
$$

We know that for every $i \in\{1,2\}$ and $j \neq i, \frac{\partial \mathfrak{a}_{i}\left(S, b r_{j}\right)}{\partial b}$ is non-positive. Following an analysis similar to above, one can argue that if the numerator of this derivative is positive, then the reference point and the bargaining frontier favor agent 2 more than they favor agent 1 ; and if so, as $b$ increases, the slope of equation (1) increases as well. Assume that this is the case, consider $b^{\prime}>b$, and write equation ( $\left.1^{\prime \prime}\right)$ by replacing $b$ with $b^{\prime}$ in equation (1). Since both lines pass from $\left(a r_{1}, a r_{2}\right)$ and equation (1) is relatively flatter, and given the shape of the bargaining frontier, we conclude that the $\left(a, b^{\prime}\right)$-solution yields a higher share to agent 2 compared to the $(a, b)$-solution. If otherwise, the converse result follows.

\subsection{Further remarks}

In this subsection, we present remarks on (i) some relevant bargaining experiments and (ii) non-cooperative support for the members of the $(\alpha, \beta)$-family

Bargaining Experiments: Hoffman and Spitzer (1982), Fischer et al. (2007), Anbarc1 and Feltovich (2013, 2018), and Bolton and Karagözoğlu (2016) all reported that the effectiveness of the disagreement point on the negotiated agreements is less than what the Nash solution or the Kalai-Smorodinsky solution predicts. Moreover, its effectiveness is influenced by the absence/presence of a norm. For instance, Anbarci and Feltovich (2018) found that if the 50-50 norm is feasible (i.e., individually rational), then the disagreement point is less effective on the negotiated agreements. In a more recent paper, Feltovich (2019) showed, in a laboratory experiment, that the bargaining agreements are more responsive to changes in the disagreement point if the disagreement point is earned (in a real effort task) rather than randomly assigned. In addition, Gächter and Riedl (2006) experimentally showed that as the salience/legitimacy of the reference point declines, its influence on the negotiated outcome and subjects' fairness judgments becomes weaker. Insights from our model have a great potential for explaining such experimental findings. Needless to say, the above-mentioned experiments were not designed to test the empirical validity of our theory. Hence, a more precise empirical test of our model would call for a new experimental design. And we leave designing and conducting such an experiment for future research.

Strategic Foundation: In the literature on bargaining problems, the so-called Nash program aims to provide strategic foundations for axiomatic solution concepts. Those studies that pursue the Nash program with a focus on the Kalai-Smorodinsky solution extensively used the axiom of IPAT (see Moulin 1984; Miyagawa 2002; Anbarc1 and Boyd 2011). More precisely, the question is studied for any normalized bargaining problem in which $d=(0, \ldots, 0)$ and $\mathfrak{a}(S, d)=(1, \ldots, 1)$ following an argument that the same results would be valid for any bargaining problem in case the positive affine transformation is appropriately chosen. Accordingly, any member of the $(\alpha, \beta)$ family can also be supported by any mechanism that supports the Kalai-Smorodinsky solution (or any particular member of the family), if one utilizes IPAT in such a way 
that the employed positive affine transformation places the (effective) salient points on $(0, \ldots, 0) \in \mathbb{R}^{n}$ and $(1, \ldots, 1) \in \mathbb{R}^{n}$.

\section{Conclusion}

We introduce two parameters that measure the influences of soft $v s$. hard power on anchor and aspiration formation into analysis of bargaining problems with a reference point. These parameters can be thought of as descriptors of the specific bargaining context, which carry information about how influential the reference point (or the disagreement point) is in shaping the (effective) anchor and the (effective) aspiration points. Alternatively, they can be interpreted as the parameters describing an arbitrator's opinion about how effectively the reference point should be utilized in reaching a settlement. As a result, we obtain a family of bargaining solutions and present characterizations of both individual members of this family as well as the whole family in bilateral bargaining problems.

As mentioned before, future work may experimentally test the validity of our theory by manipulating the salience of the reference point in simple bargaining games. Both vignettes and incentivized bargaining experiments can be used for this purpose. From a theoretical perspective, axiomatic (see Border and Segal 1997) or strategic (see Van Damme 1986) selection of the members of the $(\alpha, \beta)$-family may be of interest. Finally, our model may be used as a natural unifying framework for studying endogenous emergence of reference points in bargaining problems.

Acknowledgements We would like to thank two anonymous reviewers and the associate editor for useful comments that improved the paper. We would also like to thank Nejat Anbarci, Irem Bozbay, Thomas Demuynck, Bram Driesen, Tarik Kara, Özgür Kıbrıs, Martin Kocher, Shiran Rachmilevitch, Arno Riedl, and Ismail Sağlam for fruitful discussions and insightful comments. The usual disclaimer applies.

\section{Appendix}

Proof of Lemma 1 Take any $(S, d, r),\left(S^{\prime}, d^{\prime}, r^{\prime}\right) \in \Sigma^{2}$ such that $S \subset S^{\prime}$, ar $+(1-$ $a) d=a r^{\prime}+(1-a) d^{\prime}$, and $\mathfrak{a}(S, b r+(1-b) d)=\mathfrak{a}\left(S^{\prime}, b r^{\prime}+(1-b) d^{\prime}\right)$. Then the conditions of IND $(a, b)$-MON are satisfied for both agents. It follows for every $i \in\{1,2\}$ that $F_{i}(S, d, r) \leq F_{i}\left(S^{\prime}, d^{\prime}, r^{\prime}\right)$. Hence $F(S, d, r) \leq F\left(S^{\prime}, d^{\prime}, r^{\prime}\right)$.

Proof of Lemma 2 Take any $(S, d, r),\left(S^{\prime}, d^{\prime}, r^{\prime}\right) \in \Sigma^{2}$ such that $S \subset S^{\prime}$, ar $+(1-$ $a) d=a r^{\prime}+(1-a) d^{\prime}, b r+(1-b) d=b r^{\prime}+(1-b) d^{\prime}$, and $\mathfrak{a}(S, b r+(1-b) d)=$ $\mathfrak{a}\left(S^{\prime}, b r^{\prime}+(1-b) d^{\prime}\right)$. Then the conditions of REST $(a, b)$-MON are satisfied. Hence $F(S, d, r) \leq F\left(S^{\prime}, d^{\prime}, r^{\prime}\right)$.

Proof of Lemma 3 Take any $(S, d, r),\left(S^{\prime}, d^{\prime}, r^{\prime}\right) \in \Sigma^{2}$ such that $S=S^{\prime}$, ar $+(1-$ $a) d=a r^{\prime}+(1-a) d^{\prime}$, and $\mathfrak{a}(S, b r+(1-b) d)=\mathfrak{a}\left(S, b r^{\prime}+(1-b) d^{\prime}\right)$. Then, by REST $(a, b)$-MON, it turns out that $F(S, d, r) \leq F\left(S^{\prime}, d^{\prime}, r^{\prime}\right)$. And considering that $S^{\prime}=S$, we also have $F\left(S^{\prime}, d^{\prime}, r^{\prime}\right) \leq F(S, d, r)$. Hence $F(S, d, r)=F\left(S^{\prime}, d^{\prime}, r^{\prime}\right)$. 
Proof of Lemma 4 Take any $(S, d, r) \in \Sigma^{2}$ such that $\mathfrak{a}(S, a r+(1-a) d)=\mathfrak{a}(S, b r+$ $(1-b) d)$. Set $\left(S^{\prime}, d^{\prime}, r^{\prime}\right)=(S, a r+(1-a) d, a r+(1-a) d)$. Note that $a r+(1-a) d=$ $a r^{\prime}+(1-a) d^{\prime}$ and $\mathfrak{a}(S, b r+(1-b) d)=\mathfrak{a}\left(S, b r^{\prime}+(1-b) d^{\prime}\right)$. By LSC $(a, b)$-SP, we have $F(S, d, r)=F\left(S^{\prime}, d^{\prime}, r^{\prime}\right)$. Hence $F(S, d, r)=F(S, a r+(1-a) d$, ar + $(1-a) d)$.

Proof of Theorem 1 Fix any $(a, b) \in[0,1]^{2}$. It will be enough to prove the "only if" part of ( $i$ ) and the "if" parts of (iii) and (iv) for $b<a$ and $a \leq b$, respectively. The remaining parts follow by Lemmas $1-4$.

We first prove the "only if" part of $(i)$, which is that for any given $(a, b) \in[0,1]^{2}$, the $(a, b)$-solution satisfies WPO, SYM, IPAT, and IND $(a, b)$-MON.

Take any $(S, d, r) \in \Sigma^{2}$. For WPO, it is enough to recall that the bargaining set $S$ is convex, closed, and bounded from above. As a matter of fact, given that $n=2$, $F^{a, b}$ satisfies PO as well.

For SYM, note that the symmetric transformation $T_{\text {sym }}: \mathbb{R}^{2} \rightarrow \mathbb{R}^{2}$ maps the straight line passing through $a r+(1-a) d$ and $\mathfrak{a}(S, b r+(1-b) d)$ into the straight line $L$ passing through $T_{\text {sym }}(a r+(1-a) d)$ and $T_{\text {sym }}(\mathfrak{a}(S, b r+(1-b) d))$. Since $F^{a, b}$ satisfies PO, there cannot be a point in $L \cap T_{s y m}(S)$ that is greater than $T_{s y m}\left(F^{a, b}(S, d, r)\right)$, so it must be that $F^{a, b}\left(T_{\text {sym }}(S), T_{\text {sym }}(d), T_{\text {sym }}(r)\right)=T_{\text {sym }}\left(F^{a, b}(S, d, r)\right)$.

For IPAT, note that an affine transformation $T_{a f f}: \mathbb{R}^{2} \rightarrow \mathbb{R}^{2}(i)$ preserves the partial ordering of $\mathbb{R}^{2}$; (ii) maps straight lines into straight lines; (iii) maps ar + $(1-a) d$ into $a T_{a f f}(r)+(1-a) T_{a f f}(d)$; and $(i v)$ maps $\mathfrak{a}(S, b r+(1-b) d)$ into $\mathfrak{a}\left(T_{a f f}(S), T_{a f f}(b r+(1-b) d)\right)$. According to its definition, $F^{a, b}(S, d, r)$ is the maximum point of $S$ on the line segment connecting $\mathfrak{a}(S, b r+(1-b) d)$ and $a r+$ $(1-a) d$. Similarly, by the observations above, $F^{a, b}\left(T_{a f f}(S), T_{a f f}(d), T_{a f f}(r)\right)$ is the maximum point of $T_{a f f}(S)$ on the line segment connecting $\mathfrak{a}\left(T_{a f f}(S), T_{a f f}(b r+\right.$ $(1-b) d))$ and $a T_{a f f}(r)+(1-a) T_{a f f}(d)$. Since $T_{a f f}$ preserves the partial ordering, it must be that $F^{a, b}\left(T_{a f f}(S), T_{a f f}(d), T_{a f f}(r)\right)=T_{a f f}\left(F^{a, b}(S, d, r)\right)$.

For IND $(a, b)$-MON, without loss of generality, consider $i=1$ and $j=2$. Take any $(S, d, r),\left(S^{\prime}, d^{\prime}, r^{\prime}\right) \in \Sigma^{2}$ such that $a r+(1-a) d=a r^{\prime}+(1-a) d^{\prime}$ and $\mathfrak{a}_{2}(S, b r+(1-b) d)=\mathfrak{a}_{2}\left(S^{\prime}, b r^{\prime}+(1-b) d^{\prime}\right)$. Assume that for every $x \in S$ : $\mathfrak{a}_{1}(S, x) \leq \mathfrak{a}_{1}\left(S^{\prime}, x\right)$. For notational convenience, we let

$$
\begin{aligned}
& \bar{a}=a r+(1-a) d, \\
& \bar{b}=b r+(1-b) d, \text { and } \\
& \tilde{b}=b r^{\prime}+(1-b) d^{\prime} .
\end{aligned}
$$

Let $L$ be the straight line passing through $\bar{a}$ and $\mathfrak{a}(S, \bar{b})$. By definition of the $(a, b)$ solution, we have

$$
F^{a, b}(S, d, r)=\max \{\lambda \bar{a}+(1-\lambda) \mathfrak{a}(S, \bar{b}) \in S \mid \lambda \in[0,1]\} .
$$

That is, $F^{a, b}(S, d, r)$ is the maximal element of $L \cap S$. Let $L^{\prime}$ be the straight line passing through $\bar{a}$ and $\mathfrak{a}\left(S^{\prime}, \tilde{b}\right)$. Consider $L^{\prime} \cap S$, and set

$$
\bar{x}=\max \left\{\lambda \bar{a}+(1-\lambda) \mathfrak{a}\left(S^{\prime}, \tilde{b}\right) \in S \mid \lambda \in[0,1]\right\} .
$$


Moreover, since $\mathfrak{a}_{1}(S, \bar{b}) \leq \mathfrak{a}_{1}\left(S^{\prime}, \tilde{b}\right)$ and $\mathfrak{a}_{2}(S, \bar{b})=\mathfrak{a}_{2}\left(S^{\prime}, \tilde{b}\right)$, we have

$$
F_{1}^{a, b}(S, d, r) \leq \bar{x}_{1}
$$

By definition, $F^{a, b}\left(S^{\prime}, d^{\prime}, r^{\prime}\right)$ is the maximal element of $L^{\prime} \cap S^{\prime}$. That is,

$$
F^{a, b}\left(S^{\prime}, d^{\prime}, r^{\prime}\right)=\max \left\{\lambda \bar{a}+(1-\lambda) \mathfrak{a}\left(S^{\prime}, \tilde{b}\right) \in S^{\prime} \mid \lambda \in[0,1]\right\} .
$$

By convexity and comprehensiveness, the fact that $\mathfrak{a}_{1}(S, \bar{x}) \leq \mathfrak{a}_{1}\left(S^{\prime}, \bar{x}\right)$ implies $\bar{x} \in S^{\prime}$. It follows that the maximal element of $L^{\prime} \cap S^{\prime}$ is not less than $\bar{x}$; i.e., $\bar{x} \leq F^{a, b}\left(S^{\prime}, d^{\prime}, r^{\prime}\right)$. Therefore, $F_{1}^{a, b}(S, d, r) \leq F_{1}^{a, b}\left(S^{\prime}, d^{\prime}, r^{\prime}\right)$.

Conversely, we first focus on the "if" part of (iii). Assume that $b<a$ and take any solution $F: \Sigma^{2} \rightarrow \mathbb{R}^{2}$ satisfying all of the axioms in (iii). Take any $(S, d, r) \in \Sigma^{2}$. By IPAT, there is no generality lost by assuming that

$$
a r+(1-a) d=(0,0) \text { and } \mathfrak{a}(S, b r+(1-b) d)=(1,1) .
$$

Notice that $F_{1}^{a, b}(S, d, r)=F_{2}^{a, b}(S, d, r)$. Without loss of generality, assume that $r$ is below the 45-degree line. Consider the horizontal lines passing through $r, d$, and $b r+(1-b) d$; and take their intersections with the 45-degree line. Respectively, let these intersections be called $\tilde{r}, \tilde{d}$, and $\tilde{b}$. Notice that $a \tilde{r}+(1-a) \tilde{d}=a r+(1-a) d$ and $b \tilde{r}+(1-b) \tilde{d}=\tilde{b}$. Then let $S^{\prime \prime}$ be the convex and comprehensive hull of the points $\left(1, \tilde{b}_{2}\right),\left(\tilde{b}_{1}, 1\right)$, and $F^{a, b}(S, d, r)$. By WPO and SYM, we have

$$
F\left(S^{\prime \prime}, \tilde{d}, \tilde{r}\right)=F^{a, b}(S, d, r)
$$

Also define $S^{\prime}=\{x \in S \mid x \leq(1,1)\}$. Since $S^{\prime \prime} \subset S^{\prime} \subset S$ and $\mathfrak{a}\left(S^{\prime \prime}, \tilde{b}\right)=\mathfrak{a}\left(S^{\prime}, \tilde{b}\right)$, it follows by $b$-REST $(a, b)$-MON that

$$
F\left(S^{\prime \prime}, \tilde{d}, \tilde{r}\right) \leq F\left(S^{\prime}, \tilde{d}, \tilde{r}\right)
$$

Since $F\left(S^{\prime}, \tilde{d}, \tilde{r}\right) \in S^{\prime}$ and $F^{a, b}(S, d, r) \in P O\left(S^{\prime}\right)$, we conclude that

$$
F\left(S^{\prime \prime}, \tilde{d}, \tilde{r}\right)=F\left(S^{\prime}, \tilde{d}, \tilde{r}\right)=F^{a, b}(S, d, r) .
$$

By construction, we also have $\mathfrak{a}\left(S^{\prime}, b r+(1-b) d\right)=\mathfrak{a}\left(S^{\prime}, \tilde{b}\right)$. Then, by LSC $(a, b)$-SP,

$$
F\left(S^{\prime}, d, r\right)=F\left(S^{\prime}, \tilde{d}, \tilde{r}\right)=F^{a, b}(S, d, r)
$$

And by $b$-REST $(a, b)$-MON, we conclude that $F(S, d, r)=F^{a, b}(S, d, r)$.

Finally, assume that $a \leq b$ and take any solution $F: \Sigma^{2} \rightarrow \mathbb{R}^{2}$ satisfying all of the axioms in $(i v)$. Take any $(S, d, r) \in \Sigma^{2}$. By IPAT, there is no generality lost by assuming that

$$
a r+(1-a) d=(0,0) \text { and } \mathfrak{a}(S, b r+(1-b) d)=(1,1) .
$$


Notice that $F_{1}^{a, b}(S, d, r)=F_{2}^{a, b}(S, d, r)$. Without loss of generality, assume that $r$ is below the 45-degree line. Consider the horizontal lines passing through $r, d$, and $b r+(1-b) d$; and take their intersections with the 45-degree line. Respectively, let these intersections be called $\bar{r}, \bar{d}$, and $\bar{b}$. Notice that $a r+(1-a) d=a \bar{r}+(1-a) \bar{d}$ and $b \bar{r}+(1-b) \bar{d}=\bar{b}$. Then let $S^{\prime \prime}$ be the convex and comprehensive hull of the points $\left(1, \bar{b}_{2}\right),\left(\bar{b}_{1}, 1\right)$, and $F^{a, b}(S, d, r)$. By WPO and SYM, we have

$$
F\left(S^{\prime \prime}, \bar{d}, \bar{r}\right)=F^{a, b}(S, d, r)
$$

Also define $S^{\prime}=\{x \in S \mid x \leq(1,1)\}$. Since $S^{\prime \prime} \subset S^{\prime} \subset S$ and and $\mathfrak{a}\left(S^{\prime \prime}, \bar{b}\right)=$ $\mathfrak{a}\left(S^{\prime}, \bar{b}\right)$, it follows by $b$-REST $(a, b)$-MON that

$$
F\left(S^{\prime \prime}, \bar{d}, \bar{r}\right) \leq F\left(S^{\prime}, \bar{d}, \bar{r}\right)
$$

Since $F\left(S^{\prime}, \bar{d}, \bar{r}\right) \in S^{\prime}$ and $F^{a, b}(S, d, r) \in P O\left(S^{\prime}\right)$, we conclude that

$$
F\left(S^{\prime \prime}, \bar{d}, \bar{r}\right)=F\left(S^{\prime}, \bar{d}, \bar{r}\right)=F^{a, b}(S, d, r)
$$

By construction, we also have $\mathfrak{a}\left(S^{\prime}, b r+(1-b) d\right)=\mathfrak{a}\left(S^{\prime}, a r+(1-a) d\right)=\mathfrak{a}\left(S^{\prime}, \bar{b}\right)$. Then, by REDT $(a, b)$-SP,

$F\left(S^{\prime}, d, r\right)=F\left(S^{\prime}, a r+(1-a) d, a r+(1-a) d\right)=F\left(S^{\prime}, \bar{d}, \bar{r}\right)=F^{a, b}(S, d, r)$

And by $b$-REST $(a, b)$-MON, we conclude that $F(S, d, r)=F^{a, b}(S, d, r)$. This completes the proof.

Proof of Theorem 2 For part $(i)$, take any solution $F: \Sigma^{2} \rightarrow \mathbb{R}^{2}$ satisfying all four axioms. Then, there exists some $\left(a^{*}, b^{*}\right) \in[0,1]^{2}$ such that $F$ satisfies $\operatorname{IND}\left(a^{*}, b^{*}\right)$ MON. From Theorem 1 , it is then clear that $F$ is the $\left(a^{*}, b^{*}\right)$-solution. The converse case is trivial since any member of the $(\alpha, \beta)$-family satisfies the first three axioms as well as IND $(a, b)$-MON for some $(a, b) \in[0,1]^{2}$. Part ( $\left.i i\right)$ can be proved following similar arguments.

\section{References}

Alós-Ferrer C, García-Segarra J, Ginés-Vilar M (2018) Anchoring on Utopia: a generalization of the KalaiSmorodinsky solution. Econ Theory Bull 6:141-155

Anbarcı N (1995) Reference functions and balanced concessions in bargaining. Can J Econ 28:675-682

Anbarcı N, Boyd JH III (2011) Nash demand game and the Kalai-Smorodinsky solution. Games Econ Behav 71:14-22

Anbarc1 N, Feltovich N (2013) How sensitive are bargaining outcomes to changes in disagreement payoffs? Exp Econ 16:560-596

Anbarc1 N, Feltovich N (2018) How fully do people exploit their bargaining position? The effects of bargaining institution and the 50-50 norm. J Econ Behav Organ 145:320-334

Balakrishnan PV, Gómez JC, Vohra RV (2011) The tempered aspirations solution for bargaining problems with a reference point. Math Soc Sci 62:144-150

Bartling B, Schmidt KM (2015) Reference points, social norms, and fairness in contract renegotiation. J Eur Econ Assoc 13:98-129 
Bazerman MH (1985) Norms of distributive justice in interest arbitration. Ind Labor Relat Rev 38:558-570

Bolton GE, Karagözoğlu E (2016) On the influence of hard leverage in a soft leverage bargaining game: the importance of credible claims. Games Econ Behav 99:164-179

Border KC, Segal U (1997) Preferences over solutions to the bargaining problem. Econometrica 65:1-18

Brito DL, Buoncristiani A, Intriligator MD (1977) A new approach to the Nash bargaining problem. Econometrica 45:1163-1172

Crusius J, van Horen F, Mussweiler T (2012) Why process matters: a social cognition perspective on economic behavior. J Econ Psychol 33:677-685

Feltovich N (2019) Is earned bargaining power more fully exploited? Working paper

Fischer S, Güth W, Pull K (2007) Is there as-if bargaining? J Socio-Econ 36:546-560

Gächter S, Riedl A (2005) Moral property rights in bargaining with infeasible claims. Manag Sci 51:249-263

Gächter S, Riedl A (2006) Dividing justly in bargaining problems with claims. Soc Choice Welf 27:571-594

Gupta S, Livne ZA (1988) Resolving a conflict situation with a reference outcome: An axiomatic model. Management Science 34:1303-1314

Gupta S, Livne ZA (1989) Testing the emergence and effect of the reference outcome in an integrative bargaining situation. Mark Lett 1:103-112

Haake C-J, Qin C-Z (2018) On unification of solutions to the bargaining problem. Paderborn University, Center for International Economics, Working paper \# 113

Herweg F, Schmidt KM (2013) Loss aversion and inefficient renegotiation. Rev Econ Stud 82:297-332

Hoffman E, Spitzer ML (1982) The Coase theorem: some experimental tests. J Law Econ 25:73-98

Holm H, Runnemark E (2014) Equal splits or reference prices-behavioral differences in focal point selection. Working paper, Lund University

Irlenbusch B, Hennig-Schmidt H, Rilke MR, Walkowitz G (2017) Asymmetric outside options in ultimatum bargaining: a systematic analysis. Int J Game Theory 47:301-329

Kalai E (1977) Proportional solutions to bargaining situations: interpersonal utility comparisons. Econometrica 45:1623-1630

Kalai E, Smorodinsky M (1975) Other solutions to Nash's bargaining problem. Econometrica 43:513-518

Karagözoğlu E, Riedl A (2015) Performance information, production uncertainty, and subjective entitlements in bargaining. Manag Sci 61:2611-2626

Karagözoğlu E, Kocher MG (2018) Bargaining under time pressure from deadlines. Exp Econ (forthcoming)

Kristensen H, Gärling T (2000) Anchor points, reference points, and counteroffers in negotiations. Group Decis Negot 9:493-505

Miyagawa E (2002) Subgame-perfect implementation of bargaining solutions. Games Econ Behav 41:292308

Moulin H (1984) Implementing the Kalai-Smorodinsky bargaining solution. J Econ Theory 33:32-45

Nash JF (1950) The bargaining problem. Econometrica 18:155-162

Raiffa H (1953) Arbitration schemes for generalized two-person games. In: Kuhn HW, Tucker AW (eds) Annals of Mathematical Studies, 28: 361-387. Princeton University Press, Princeton

Roemer J (1986) The mismarriage of bargaining theory and distributive justice. Ethics 97:88-110

Rosenthal RW (1976) An arbitration model for normal-form games. Math Oper Res 1:82-88

Sebenius JK (1992) Negotiation analysis: a characterization and review. Manag Sci 38:18-38

Thomson W (1981) A class of solutions to bargaining problems. J Econ Theory 25:431-441

Van Damme E (1986) The Nash bargaining solution is optimal. J Econ Theory 38:78-100

Yockey MD, Kruml SM (2009) Everything is relative, but relative to what? Defining and identifying reference points. J Bus Manag 15:95-109

Publisher's Note Springer Nature remains neutral with regard to jurisdictional claims in published maps and institutional affiliations. 